\title{
Non-Bacterial Thrombotic Endocarditis: A Presentation of COVID-19
}

\author{
Dilan Balata ${ }^{1}$, Johan Mellergård², David Ekqvist ${ }^{3}$, Jacek Baranowski ${ }^{4}$, Isidro Albert Garcia ${ }^{5}$, Marina Volosyraki ${ }^{6}$, Mats Broqvist $^{1}$ \\ ${ }^{1}$ Department of Cardiology, University Hospital Linköping, Linköping, Sweden \\ ${ }^{2}$ Department of Neurology, University Hospital Linköping, Linköping, Sweden \\ ${ }^{3}$ Department of Infectious Diseases, University Hospital Linköping, Linköping, Sweden \\ ${ }^{4}$ Department of Clinical Physiology, University Hospital Linköping, Linköping, Sweden \\ ${ }^{5}$ Department of Radiology, University Hospital Linköping, Linköping, Sweden \\ ${ }^{6}$ Department of Acute Internal Medicine and Geriatrics, University Hospital Linköping, Linköping, Sweden
}

Received: $16 / 06 / 2020$

Accepted: $24 / 06 / 2020$

Published: $21 / 07 / 2020$

How to cite this article: Balata D, Mellergård J Ekqvist D, Baranowski J Garcia IA, Volosyraki M, Broqvist M. Non-bacterial thrombotic endocarditis: a presentation of COVID-19. EJCRIM 2020;7: doi:10.12890/2020_001811.

Conflicts of Interests: The Authors declare that there are no competing interests.

Acknowledgements: We thank Charalampos Georgiopoulos, MD, PhD, Department of Radiology at Linköping University Hospital for providing the MRI images and corresponding interpretation.

This article is licensed under a Commons Attribution Non-Commercial 4.0 License

\section{ABSTRACT}

The SARS-CoV-2 virus is a newly emergent pathogen first identified in Wuhan, China, and responsible for the COVID-19 global pandemic. In this case report we describe a manifestation of non-bacterial thrombotic endocarditis with continuous peripheral embolization in a COVID19-positive patient. The patient responded well to high-dose LMWH treatment with cessation of the embolic process.

\section{LEARNING POINTS}

- Raising awareness of possible complications of COVID-19.

- To highlight the importance of the careful consideration of and dosage of anticoagulation in non-bacterial thrombotic endocarditis.

\section{KEYWORDS}

Endocarditis, mitral valve, ultrasound, COVID-19

\section{CASE PRESENTATION}

A 76-year-old woman with arterial hypertension and gastroesophageal reflux disease presented to the emergency department with acute onset right-sided central facial palsy, dysphasia and dysarthria. A week earlier the patient had visited the local outpatient clinic with vertigo and fatigue. A weak systolic murmur was noted during auscultation. Due to the pandemic our hospital employed a rigid protocol for admission, dividing the emergency department into a regular section and a section for patients with suspected COVID-19 where testing for SARS-CoV-2 and adequate hygiene protocols were employed. No respiratory symptoms or other symptomatology was communicated at admission and the patient was admitted via the regular route. However, retrospective history taking revealed anosmia and ageusia 2 weeks before admission. Initial testing showed elevated D-dimer of $0.9 \mathrm{mg} / \mathrm{I}$ (ref $<0.31 \mathrm{mg} / \mathrm{l})$. Inflammatory parameters were all within normal ranges. 
Methods and Management

Initial work-up included a CT scan that showed a subacute infarction in the right cerebellar hemisphere; no fresh infarctions were observed. Treatment with $75 \mathrm{mg}$ acetylsalicylic acid was initiated. Cerebral angiography of the main vessels showed no signs of thrombosis or dissection. A carotid Doppler did not reveal any embolic sources or stenotic segments. Cardiac telemetry did not unmask any arrhythmias. MRI showed widespread bilateral supra and infratentorial embolic infarctions (Fig. 1). After the appearance of weakness in the right arm 4 days after admission, acetylsalicylic acid was substituted with 8,000 anti-factor Xa units of tinzaparin. Six days later a second MRI was performed due onset of weakness in the right leg, and revealed new infarctions in the left frontal lobe. Tinzaparin was increased to 10,000 anti-factor Xa IU. Trans-thoracic echocardiography (TTE) displayed no abnormalities; however, trans-esophageal echocardiography (TEE) the following day revealed two vegetations on the mitral valve, one on the anterolateral commissure (Fig. 2) and the other on the anterior leaflet. A smaller vegetation was discovered on the aortic valve. No valvular anatomic defects were observed.
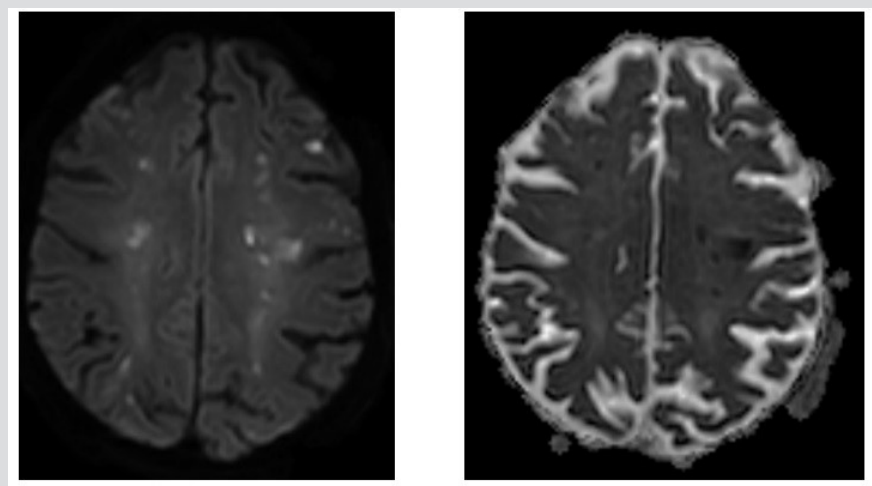

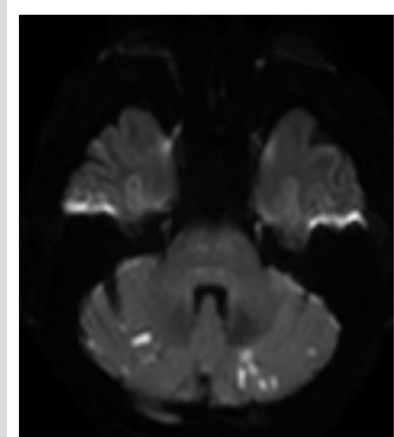

a

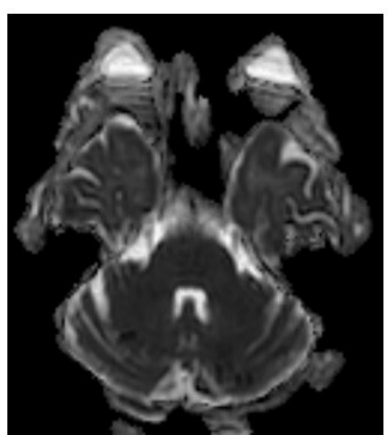

b
Figure 1. Brain MRI. (a) Diffusion weighted images (DWl; $b=1000$ ) and (b) ADC maps recorded with a 3 Tesla scanner on admission day demonstrated widespread bilateral embolic infarctions supra- and infratentorially

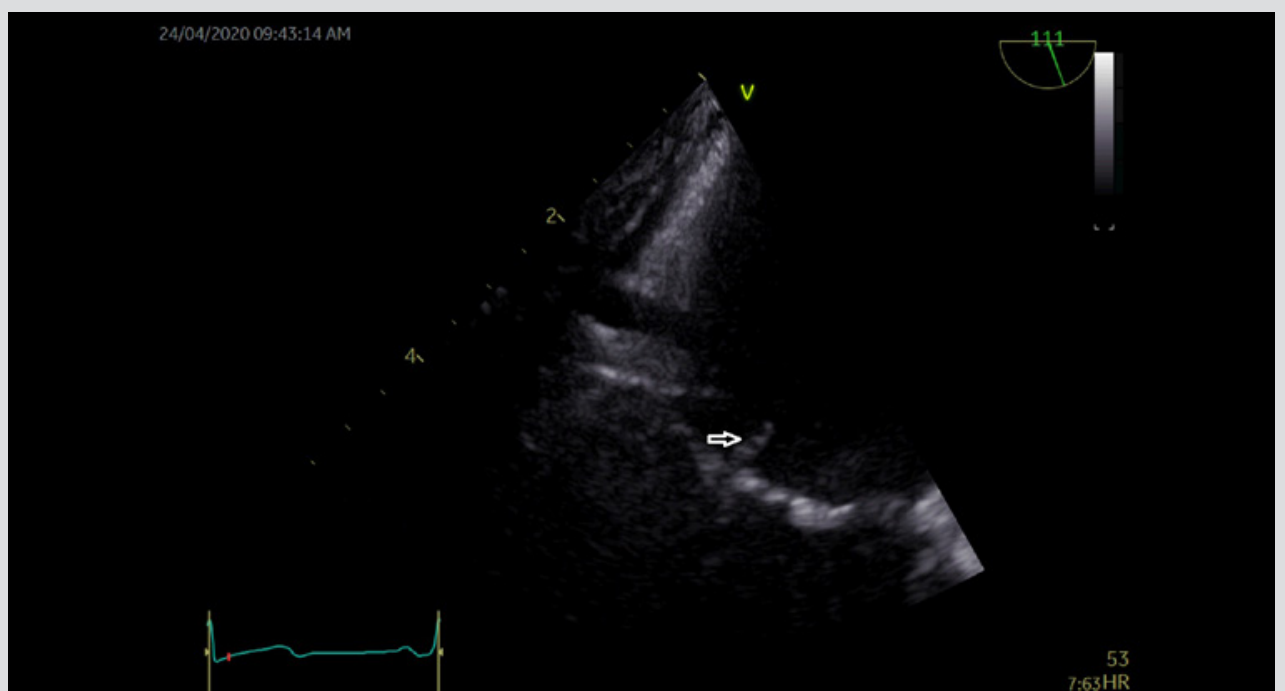

Figure 2. Trans-esophageal echography revealed two vegetations on the mitral valve, one measuring $6 \times 7 \mathrm{~mm}$ and situated basally adjacent to the anterolateral commissure (marked with an arrow) 
The patient fulfilled one major and one minor Duke criteria, denoting possible infective endocarditis. Empiric antibiotic treatment with vancomycin and cefotaxime was initiated after blood culturing. The patient still had no clinical or laboratory signs of infection which raised the suspicion of non-bacterial thrombotic endocarditis (NBTE). At this point a nasopharyngeal swab was obtained and RT-PCR testing for SARS-CoV-2 was initiated and came back positive for COVID-19. Nineteen days after admission there was progression of limb weakness in the right extremities and a final MRI showed signs of multiple new emboli. Anti-factor Xa activity measured 3 hours after subcutaneous injection at this point showed results within the therapeutic interval at 0.71 anti-factor Xa IU/ml (ref 0.6-1.0). Tinzaparin was finally raised to 12,000 anti-factor Xa IUto reach the higher end of the therapeutic interval.

Abdominal and thoracic CT as well as gynaecological examination did not reveal any solid tumours. As an incidental finding, infarctions of embolic origin and varying age were observed bilaterally in the kidneys and in the spleen. A full-body F-18 FDG PET scan showed no signs of malignancy or obvious foci of infection (Fig. 3). Odontological examination demonstrated signs of pulpitis in the first right molar.

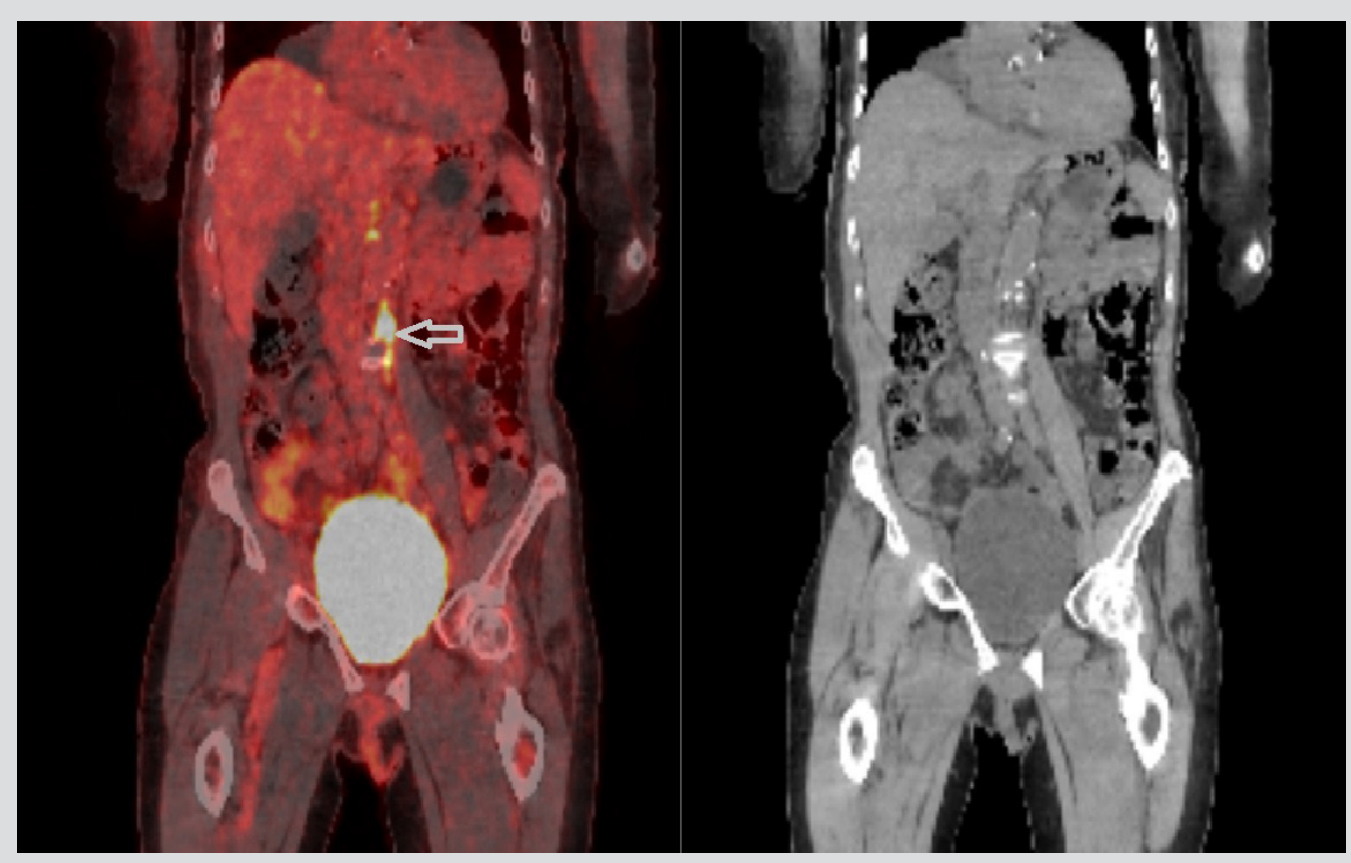

Figure 3. A full body F-18 FDG PET scan following the endocarditis protocol showed unspecific areas of increased uptake in the retroperitoneum (marked with an arrow) without enlarged lymph nodes in the area, thought to be caused by lumbar vein embolization. No signs of malignancy or obvious foci of infection were observed

Laboratory testing did not reveal coagulopathies and a full immunological panel displayed no abnormalities. Repeated blood culturing did not detect any pathogens. Laboratory tests including leukocyte cell counts and CRP were repeated several times, with CRP only being elevated at one point to $56 \mathrm{mg} / \mathrm{l}$ ( ref $<10 \mathrm{mg} / \mathrm{l}$ ), coinciding with recent embolization. Antibiotic treatment was discontinued after 4 weeks. At the time this report was submitted, the patient was still experiencing dysphasia, dysarthria, right-sided central facial palsy and hemiparesis, but there was no further progress of symptoms 6 weeks after the final increase in the tinzaparin dosage.

\section{DISCUSSION}

A case of NBTE in a previously relatively healthy 76-year old woman is described. NBTE is a form of endocarditis classically associated with malignancies and highly inflammatory conditions such as SLE ${ }^{[1,2]}$. We suspect that in this case it was secondary to a subclinical COVID-19 infection.

The course of the disease in this case correlates with classic NBTE, including the observed valve vegetations, embolization to multiple organs ${ }^{[1]}$ and no signs of infection apart from an inflamed tooth that was considered an incidental finding. No obvious malignancy or other associated conditions could be found. A full work-up with laboratory testing and a battery of radiological examinations did not reveal any conclusive aetiology.

The pathogenesis of NBTE is still uncertain. It is currently hypothesized that there is activation of interleukins and cytokines released by immune cells. This causes endothelial damage and induces a hypercoagulable state that together facilitate depositions of thrombi consisting of mononuclear cells, fibrin, immune complexes and platelets [3]. These sterile vegetations have less cellular organization at the deposition site which makes them friable and readily prone to detachment with subsequent systemic embolization ${ }^{[3]}$. 
The standard treatment for NBTE is currently full-dose LMWH or warfarin [1]. Several recent case reports have attested to the increased incidence of thromboembolic events observed in relation to COVID-19. A multicentre cohort study in the Netherlands investigated thromboembolic complications in 184 patients treated in an intensive care unit with proven COVID-19 pneumonia. A $31 \%$ incidence of thromboembolism was reported despite treatment with standard doses of thromboprophylaxis ${ }^{[4]}$.

One report describes five cases of ischaemic stroke associated with COVID-19 in patients aged under 50. These patients had concomitant respiratory symptoms, but ultrasound of the carotid arteries and heart did not reveal any embolic sources. However, information on whether TEE specifically was performed in these patients is lacking ${ }^{[5]}$. In our presented case, TTE results were normal, with only the TEE revealing the vegetations. There was continuing embolization despite full-dose LMWH treatment. This raises the point that patients with COVID-19 and NBTE might require higher than standard doses.

Based on these findings, it is possible that COVID-19 may have been the cause of the NBTE. The underlying mechanisms behind both COVID19-induced thromboembolism and NBTE appear to be similar. Both involve activation of the immune system, an inflammatory response and subsequent thrombus formation. Curiously, in this case the laboratory markers did not indicate high levels of inflammation, suggesting the possibility of there being another phenomenon underlying the increased thrombogenicity.

At our hospital we employ RT-PCR testing for COVID-19 using samples taken with a nasopharyngeal swab. This is a relatively cheap and simple method that can be utilized in all patient groups. It is important to note that currently no peer-reviewed publications have reported sensitivity and specificity. However, results are considered to have a high specificity of 96-98\% according to a non-peer reviewed study and have a high positive predictive value in a suspected patient group ${ }^{[6]}$. Nevertheless, a major drawback is that there is a relatively high rate of false-negative tests of $2-29 \%$ depending on factors such as sampling technique and timing ${ }^{[7]}$. We recommend that testing should be repeated if there is any remaining clinical suspicion.

It is important to note that upon admission the patient had no symptoms suggestive of COVID-19; however, the patient was not asked about anosmia and ageusia at that point. It should be highlighted that symptoms are highly variable, and we suggest awareness should be raised concerning SARS-CoV-2, with subtle symptoms taken into consideration. However, a blind testing policy without clinical suspicion was not logistically feasible at our hospital since laboratory equipment and testing reagent were limited. There was not sufficient manpower to run testing on such a large scale. It currently takes 8-12 hours to obtain a result, delaying diagnosis and further limiting the availability of isolation wards.

\section{CONCLUSION}

NBTE is a condition mainly caused by malignancies and autoimmune diseases. If these causes are excluded and there is no suspicion of bacterial endocarditis, we suggest that in light of this case, it might be of value to test for COVID-19 as NBTE may be one of the first manifestations. Treatment with high-dose LMWH is recommended if the patient does not respond to standard doses.

\section{REFERENCES}

1. Bauer KA, Lawrence LK, Finlay G. Nonbacterial thrombotic endocarditis. UpToDate, 2019. Available at: https://www.uptodate.com/contents/nonbacterial-thromboticendocarditis (accessed 27 May 2020).

2. Choi JH, Park JE, Kim JY, Kang T. Non-bacterial thrombotic endocarditis in a patient with rheumatoid arthritis. Korean Circ J 2016;46(3):425-428

3. Shatila W, Rizkallah A, Saad Aldin E, Tfayli A. Nonbacterial thrombotic endocarditis as the sole manifestation of stage IV gastric cancer: a case report. J Med Case Rep 2014;8(1):267.

4. Klok FA, Kruip MJHA, van der Meer NJM, Arbous MS, Gommers D, Kant KM, et al. Incidence of thrombotic complications in critically ill ICU patients with COVID-19. Thromb Res 2020;191:148?-150.

5. Oxley TJ, Mocco J, Majidi S, Kellner CP, Shoirah H, Singh IP, et al. Large-vessel stroke as a presenting feature of Covid-19 in the young. New Engl J Med 2020;382(20): 60.

6. Zitek T. The appropriate use of testing for COVID-19. West J Emerg Med 2020;21(3):470.

7. Watson J, Whiting PF, Brush JE. Interpreting a COVID-19 test result. BMJ 2020;369:m1808. 